\title{
Fuzzy vs. Probabilistic Techniques to Address Uncertainty for Radial Distribution Load Flow Simulation
}

\author{
Roma Raina', Mini Thomas ${ }^{2}$ \\ ${ }^{1}$ Manipal University, Dubai, UAE \\ ${ }^{2}$ Jamia Millia Islamia University, New Delhi, India \\ Email: roma.raina@manipaldubai.com
}

Received January 8, 2012; revised February 10, 2012; accepted February 22, 2012

\begin{abstract}
For Power distribution system the most important task for distribution engineer is to efficiently simulate the system and address the uncertainty using a suitable mathematical method. This paper presents a comparison of two methods used in analyzing uncertainties. The first method is Montecarlo simulation (MCS) that considers input parameters as random variables and second one is fuzzy alpha cut method (FAC) in which uncertain parameters are treated as fuzzy numbers with given membership functions. Both techniques are tested on a typical Load flow solution simulation, where connected loads are considered as uncertain. In order to provide a basis for comparison between above two approaches, the shapes of the membership function used in the fuzzy method is taken same as the shape of the probability density function used in the Monte Carlo simulations. For more than one uncertain input variable, simulation result indicates that MCS method provides better output results compared to FAC, however takes more time due to number of runs. FAC provides an alternate method to MCS when addressing single or limited input variables and is fast.
\end{abstract}

Keywords: Fuzzy Set; Radial Power Distribution; Montecarlo; Load Flow

\section{Introduction}

Current time power distribution systems, especially in developing countries, are steadily approaching towards its maximum operating limits and voltage stability is a major concern. Voltage instability leads to blackouts and makes the system unreliable. It is important to have a reliable power distribution system, which maintains voltages within the permissible range and ensure a high quality of output.

The voltage instability can be addressed using the various techniques e.g. reconfiguration, addition of capacitor banks etc., however need an efficient simulation of load flow and a mathematical method which address the uncertainty efficiently especially the uncertainty associated with input parameters. Distribution system uncertainties are due to error in measurement of feeder parameters, variation in expected values of the demands with time etc. and are main causes of uncertain simulation outputs.

Uncertainty can be analyzed and addressed using several techniques. In past, many solution methods have been developed on Load Flow distribution networks using Fuzzy and probabilistic models.

D. M. Falcao describes the conceptual basis and preliminary results of a load estimation based on the application of neural network and fuzzy set techniques [1]. Chi-Wen Liu presents a neurofuzzy network for voltage security monitoring [2]. I. J. Ramirez-Rosado, Dominguez-Navarro, presents a new possibilistic (fuzzy) model for the multiobjective optimal planning of power distribution networks [3]. Vikas kumar presents a comparison between probalistic and Fuzzy alpha cut techniques in general [4]. A. J Abebe presents a comparison of two methods (fuzzy alpha cut and Monte Carlo simulation) of analysis of uncertainty arising from uncertain model parameters [5]. However none have been found comparing two methods and addressing importance of each other for radial distribution system calculations.

This paper presents a comparison of "Monte-Carlo simulation method (MCS)" a technique based on probability and "Fuzzy alpha cut method (FAC)" a technique based on Fuzzy. The MCS technique treats uncertain parameter as random variable that obeys a given probabilistic distribution and model output is then a random variable. The fuzzy analysis is based on fuzzy logic and fuzzy set theory, which is widely used in representing uncertain knowledge. Uncertain model parameters are treated as fuzzy numbers with a membership function.

\section{Methodology}

\subsection{Steps \& Input Data}

For simulation purpose this paper uses a load flow algo- 
rithm, based on concept described by R. Raina, M Thomas, R. Ranjan [6]. The algorithm calculates the total real and reactive system power loss of the radial distribution network. The algorithm considers input parameters as random variables for Monte Carlo simulation and as fuzzy numbers with a given membership function for fuzzy logic.

This simulation is run on a typical 19 bus distribution system from the D. Thukram, H. M W. Banda and J. Jerome [7] shown in Figure 1.

Input connected load data for the feeder are given in Table 1, Conductor data for the feeders are given in Table 2 and Table 3.

Figure 2 shows the Typical Load Flow calculation chart used for Fuzzy and Monte Carlo simulation. The details of formulas and computing method are in [6].

\subsection{Monte-Carlo Simulation Method (MCS)}

The MCS principle is described in Figure 3. Uncertain input parameter is considered as a random variable $\mathrm{P}$ and numbers of realizations $P_{i}$ of $P$ are generated and load flow algorithm is run for each of them producing an output $R_{i}$. The set of outputs $R_{i}$ represents the set of realizations of the random variable $\mathrm{R}$. The statistical properties of $\mathrm{R}$ are therefore computed from the realizations $\mathrm{R}_{\mathrm{i}}$.

\subsection{Fuzzy Alpha-Cut Method (FAC)}

This method uses fuzzy set theory to represent uncertainty or imprecision in the parameters. Uncertain parameters are considered to be fuzzy numbers with some membership functions. In fuzzy logic, it represents the degree of truth as an extension of valuation. Degrees of truth are often confused with probabilities, although they are con-

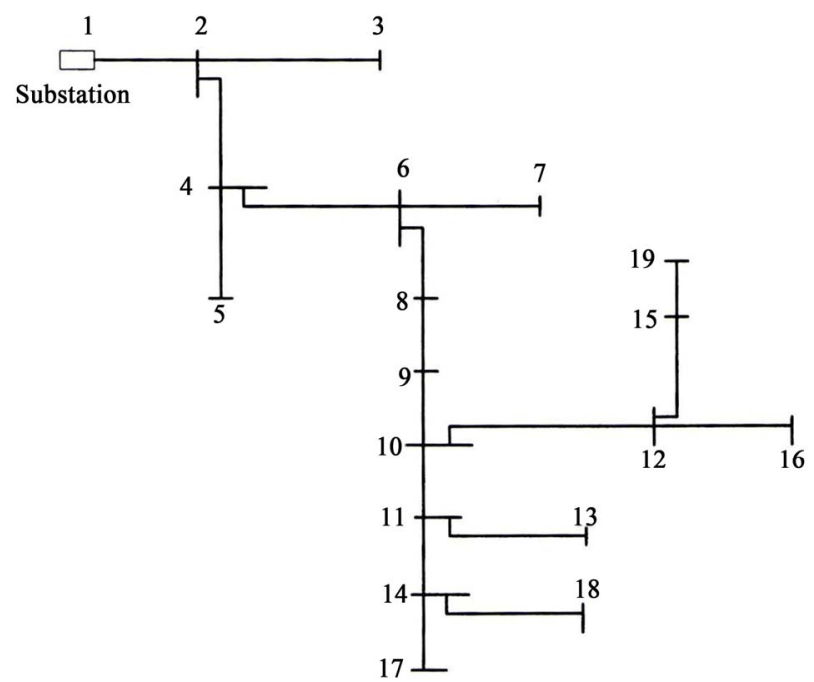

Figure 1. Shows a practical 19 bus distribution feeder used for the modeling and simulation purpose.
Table 1. Load data.

\begin{tabular}{cccc}
\hline \multirow{2}{*}{ Node } & \multicolumn{3}{c}{ Phase Load in kVA } \\
\cline { 2 - 4 } & A & B & C \\
\hline 2 & 64 & 32 & 64 \\
3 & 68 & 32 & 60 \\
4 & 25 & 35 & 40 \\
5 & 40 & 32 & 28 \\
6 & 26 & 19 & 18 \\
7 & 60 & 50 & 50 \\
8 & 46 & 33 & 21 \\
9 & 76 & 92 & 82 \\
10 & 21 & 26 & 16 \\
11 & 46 & 46 & 68 \\
12 & 60 & 50 & 50 \\
13 & 27 & 33 & 40 \\
14 & 19 & 19 & 25 \\
15 & 27 & 30 & 43 \\
16 & 48 & 64 & 48 \\
17 & 40 & 30 & 30 \\
18 & 33 & 33 & 34 \\
19 & 54 & 62 & 44 \\
\hline
\end{tabular}

Table 2. Conductor data.

\begin{tabular}{ccc}
\hline Conductor type & Resistance PU/Km & Reactance PU/Km \\
\hline 1 & 0.008600 & 0.003700 \\
2 & 0.012950 & 0.003680 \\
\hline
\end{tabular}

Table 3. Conductor code $\&$ distances.

\begin{tabular}{cccc}
\hline $\begin{array}{c}\text { Sending End } \\
\text { Node(IR) }\end{array}$ & $\begin{array}{c}\text { Receiving End } \\
\text { Node(IR) }\end{array}$ & Conductor Code & Distance in Km \\
\hline 1 & 2 & 1 & 3 \\
2 & 3 & 2 & 5 \\
2 & 4 & 1 & 1.5 \\
4 & 5 & 2 & 1.5 \\
4 & 6 & 1 & 1 \\
6 & 7 & 2 & 2 \\
6 & 8 & 1 & 2.5 \\
8 & 9 & 1 & 3 \\
9 & 10 & 1 & 5 \\
10 & 11 & 1 & 1.5 \\
10 & 12 & 1 & 1 \\
11 & 13 & 2 & 5 \\
11 & 14 & 1 & 3.5 \\
12 & 15 & 1 & 4 \\
12 & 16 & 2 & 1.5 \\
14 & 17 & 1 & 6 \\
14 & 18 & 2 & 5 \\
15 & 19 & 1 & \\
\hline & & & 4 \\
\hline
\end{tabular}




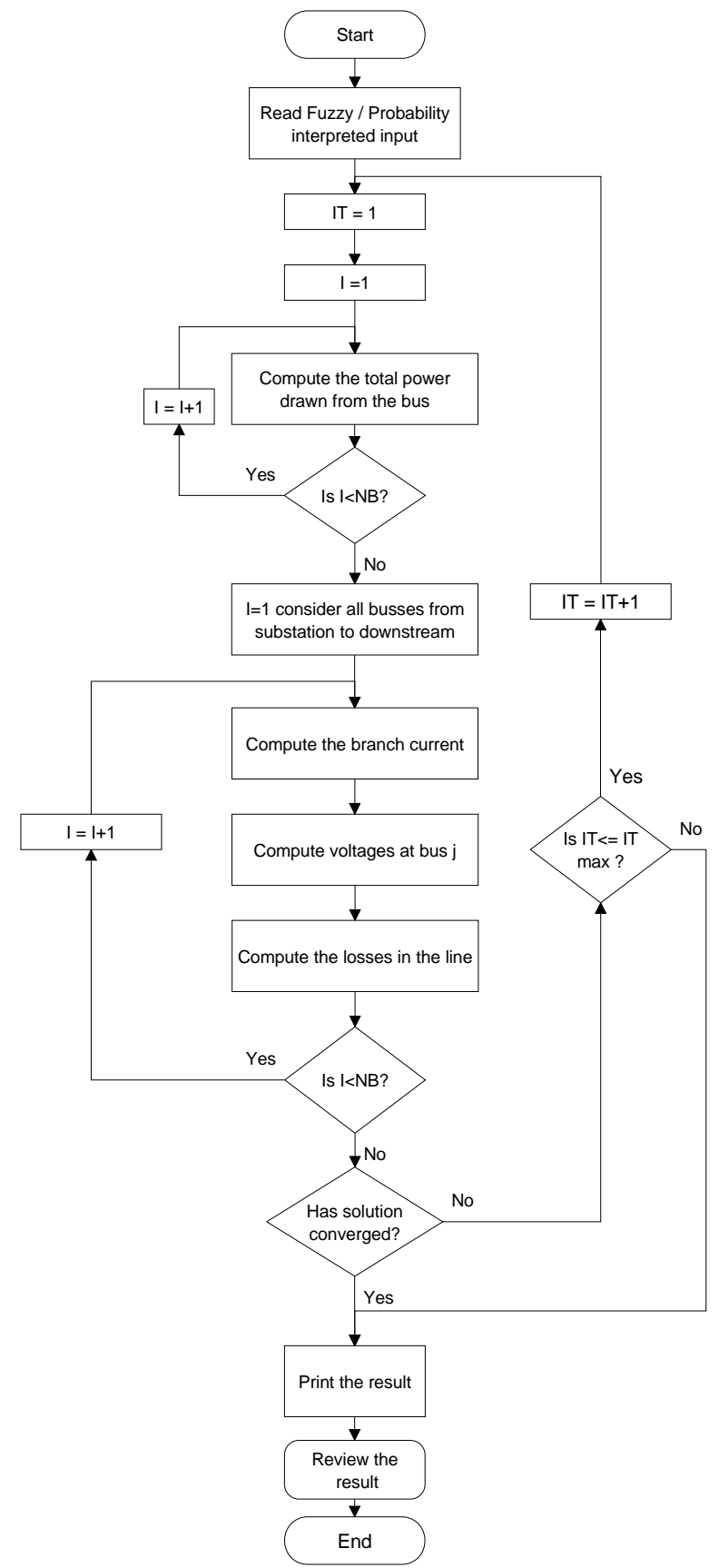

Figure 2. Typical Fuzzy \& Monty Carlo Load Flow calculation chart used with fuzzy/probability interpreted inputs.

ceptually distinct, because fuzzy truth represents membership in vaguely defined sets, not likelihood of some event or condition. Figure 4 shows a parameter $\mathrm{P}$ represented as a triangular fuzzy number with support of $\mathrm{A}_{0}$. The wider the support of the membership function, the higher the uncertainty. The fuzzy set that contains all elements with a membership of $\alpha \varepsilon[0,1]$ and above is called the $\alpha$-cut of the membership function. At a resolution level of $\alpha$, it will have support of $\mathrm{A}_{\alpha}$. Higher the value of $\alpha$, higher the confidence in the parameter.

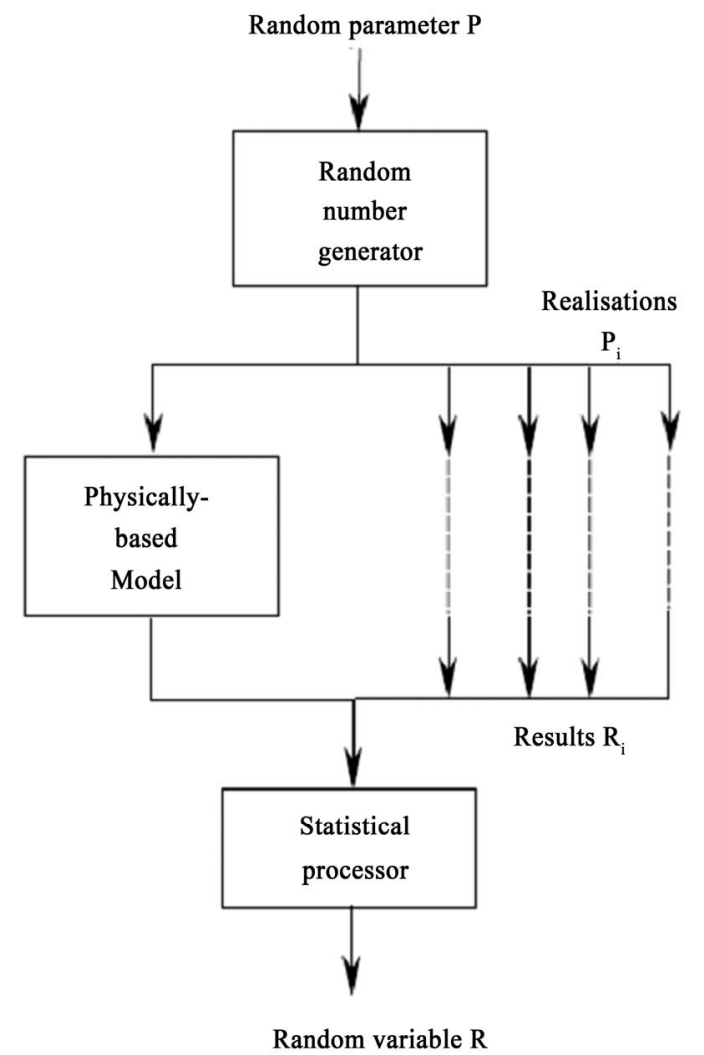

Figure 3. Sketch for Monte Carlo Simulation method.

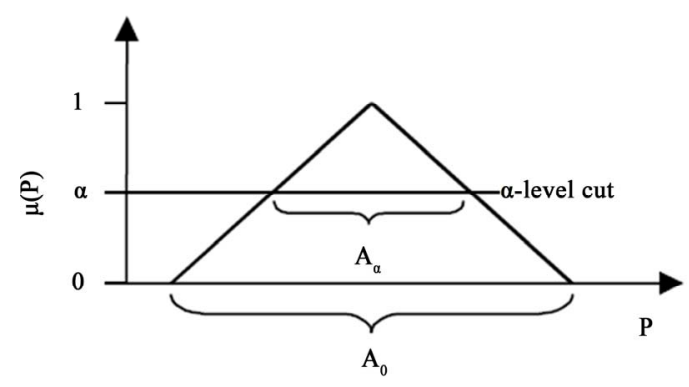

Figure 4. Fuzzy representation and Alpha cut.

The method is based on the extension principle, which implies that functional relationships can be extended to involve fuzzy arguments and can be used to map the dependent variable as a fuzzy set. The membership function is cut horizontally at a finite number of $\alpha$-levels between 0 and 1 . For each $\alpha$-level of the parameter, the model is run to determine the minimum and maximum possible values of the output. This information is then directly used to construct the corresponding fuzziness (membership function) of the output which is used as a measure of uncertainty.

In order to provide a uniform basis of comparison between two techniques, the shapes of membership Function (MF) used in fuzzy method is assumed same as shape of probability density function used for Monte Carlo simulation. 
Simulation uses an assumption that connected load will vary between $20 \%$ to $135 \%$ of rated data and are shown in Figure 5 and Figure 6. Above assumption can be represented by a triangular distribution.

\section{Simulation Results}

In order to assess the results, two analyses were carried out: a spatial analysis, for which a measure of uncertainty was devised, and a point wise analysis, where the cumulative density probability function (CDF) for the MCS methods and the membership function for the FAC method of the output values were analysed.

\subsection{Spatial Analysis}

To evaluate the spatial distribution of uncertainty, measure of uncertainty for the two methods is established. For the MCS method, such a measure is defined by the ratio of the standard deviation to the mean value, and for the FAC method the ratio of the 0.1-level support to the value for the membership function is equal to 1 (Refer Figure 7).

\section{Fuzzy Membership function representation}

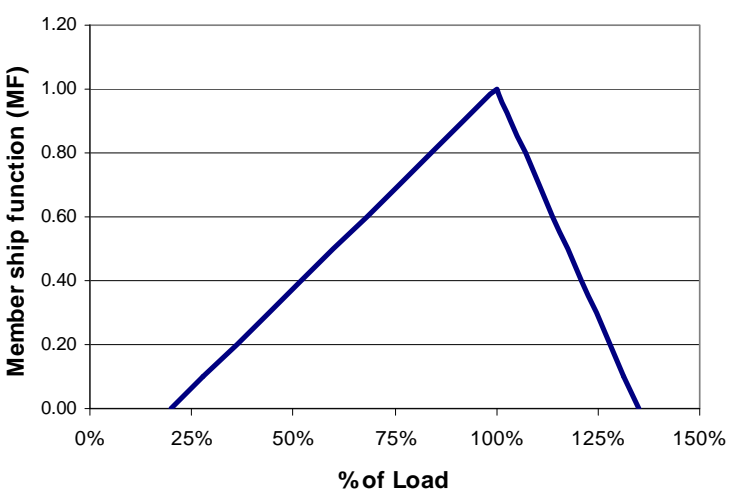

Figure 5. Fuzzy Membership function (MF) representation.

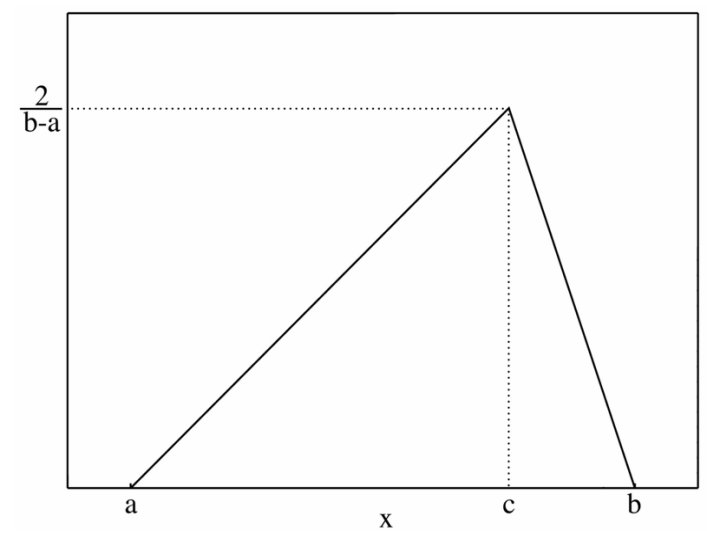

Figure 6. Probability density function representing limit a (20\% in our case), upper limit b $(130 \%$ on our case) and mode $\mathrm{c}(\mathbf{1 0 0 \%})$, where $\mathrm{a}<\mathrm{b}$ and $\mathrm{a} \leq \mathrm{c} \leq \mathrm{b}$.
Table 4 shows the calculated uncertainty values with FAC and MCS methods. It may be noted that uncertainty measure with FAC is much higher than that calculated using MCS method.

For understating this further, the output results are plotted for all the trails. Figure 8 is the histogram of system power losses for 500 trails using MCS method and Figure 9 is the scatter plot of MF vs. system power loss using FAC method. The output result range (variance) observed using FAC technique is much higher than that obtained from MCS method. It can be concluded that MCS method provides more reliable results compared to FAC method with less variance or uncertainty.

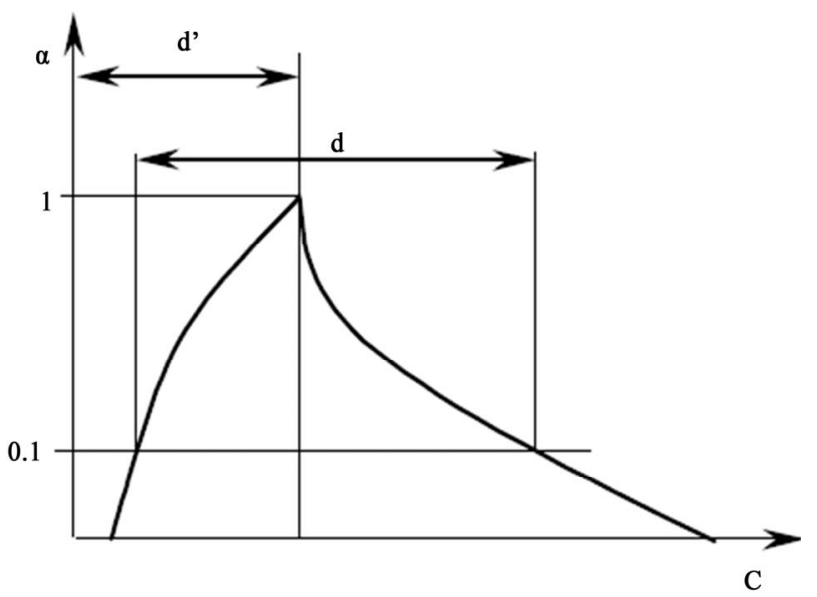

Figure 7. Measure of uncertainty for FAC technique.

Table 4. Calculated uncertainty values.

\begin{tabular}{ccc}
\hline Method & Basis (3.1) & Uncertainty value \\
\hline FAC & $\begin{array}{l}\text { Ratio of the 0.1-level support } \\
\text { to the value at MF equal to 1 }\end{array}$ & 1.779 \\
MCS & $\begin{array}{c}\text { Ratio of the Standard Deviation } \\
\text { to the Mean Value }\end{array}$ & 0.315 \\
\hline
\end{tabular}

Histogram of Feeder Total Real Loss \& Reactive Loss for $\mathbf{5 0 0}$ random trail

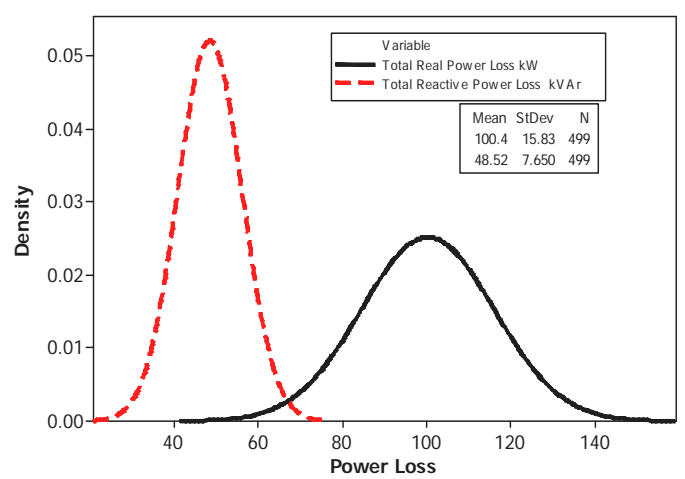

Figure 8. Total real \& reactive power loss for 500 trails. 


\subsection{Pointwise Analysis}

To evaluate the result obtained from spatial analysis further a point wise analysis is carried out and the algorithm is run for three cases shown in Table $\mathbf{5}$.

Figures 10-13 show the normalized Results obtained

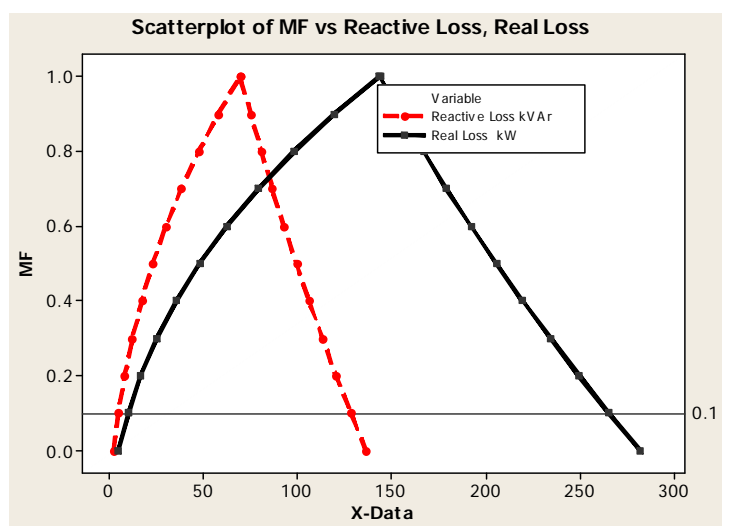

Figure 9. Scatter plot of MF vs. reactive, real power loss.

Table 5. Calculated uncertainty values.

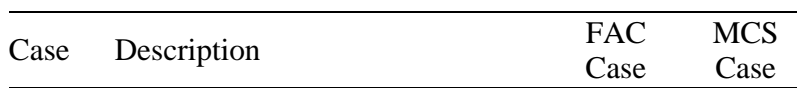

All connected load considered

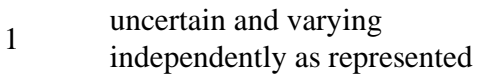

F-1 R-1

by Figure 5 and 6

Only one connected load connected to node 9 is assumed

$2 \quad$ as uncertain and varying independently as represented

F-2 R-2

by Figure 5 and $\mathbf{6}$

Three connected load connected to node 7, 9 \& 12 are assumed as uncertain and varying independently as represented by Figure 5 and 6

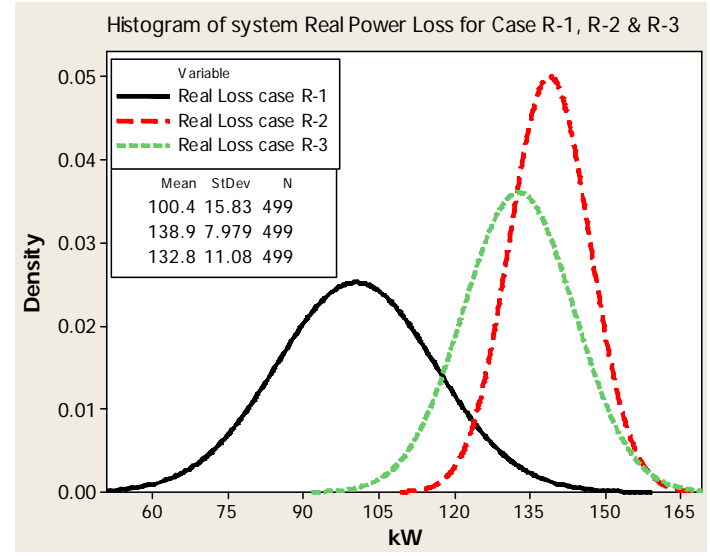

Figure 10. System real power loss for case R-1, R-2 \& R-3. shows that output range (variance) is more for Case 1, followed by Case 3 and minimum for Case 2 for each individual method. However when output is compared for two methods, the output from FAC method shows much more variance magnitude as compared to what obtained from MCS technique.

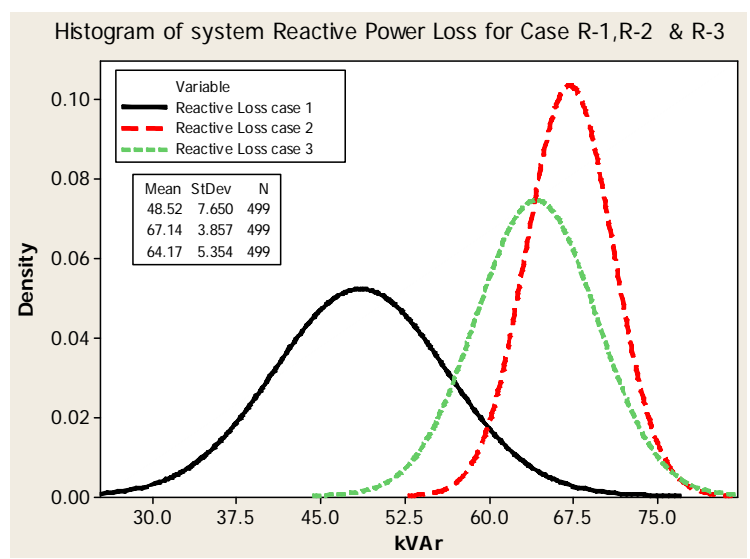

Figure 11. System reactive power loss for case R-1, R-2 \& R-3.

Histogram of system Real Power Loss for Case F-1,F-2 \& F-3

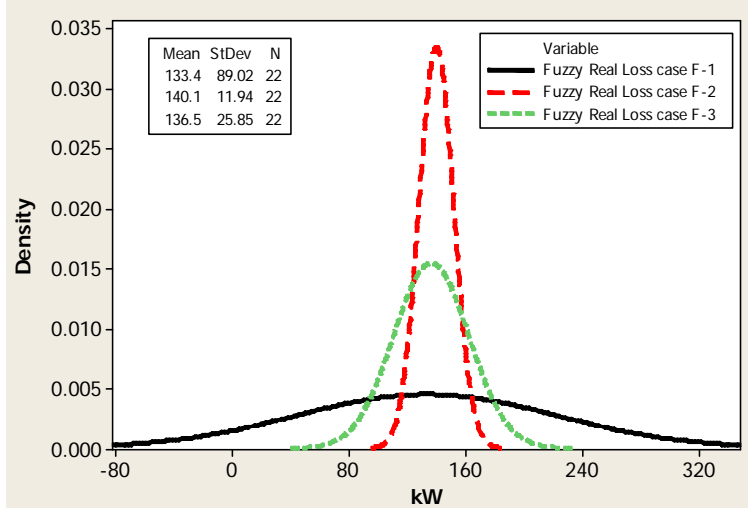

Figure12. System real power loss for case F-1, F-2 \& F-3.

Histogram of System Reactive Power Loss for Cases F-1,F-2 \& F-3

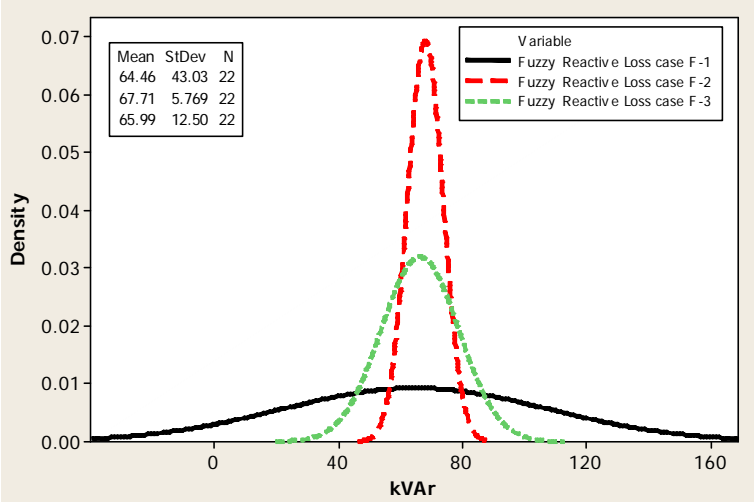

Figure 13. System reactive power loss for cases F-1, F-2 \& F-3. 
Figure 14-16 show the cumulative density function (CDF) and normalized integrated fuzzy number plot for defined three cases. Results are comparable for Case 2 but the variance increases for Case 3 and is maximum for Case 1 . The results also shows that variance difference between two techniques depends upon the number of uncertain input variables considered.

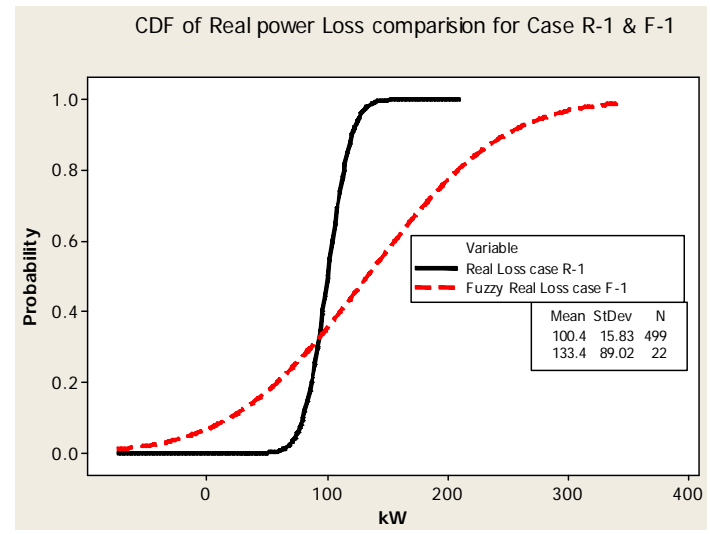

Figure 14. CDF of real power loss comparison for case R-1 \& F-1.

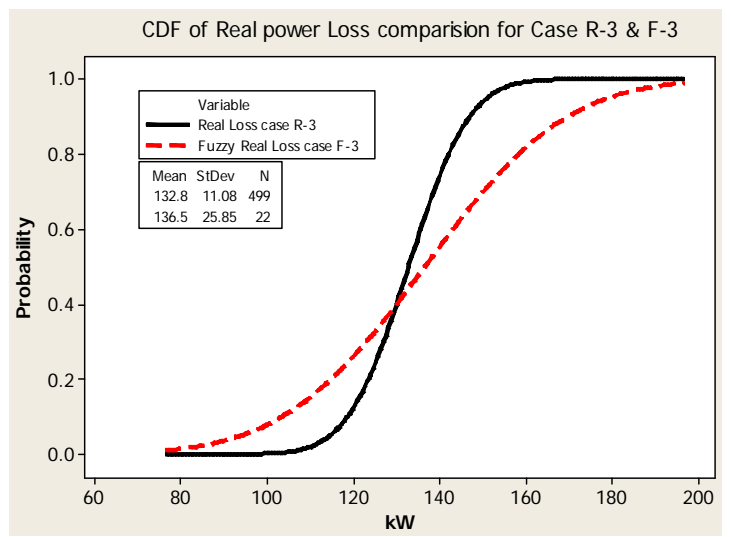

Figure 15 CDF of real power loss comparison for case R-3 \& F-3.

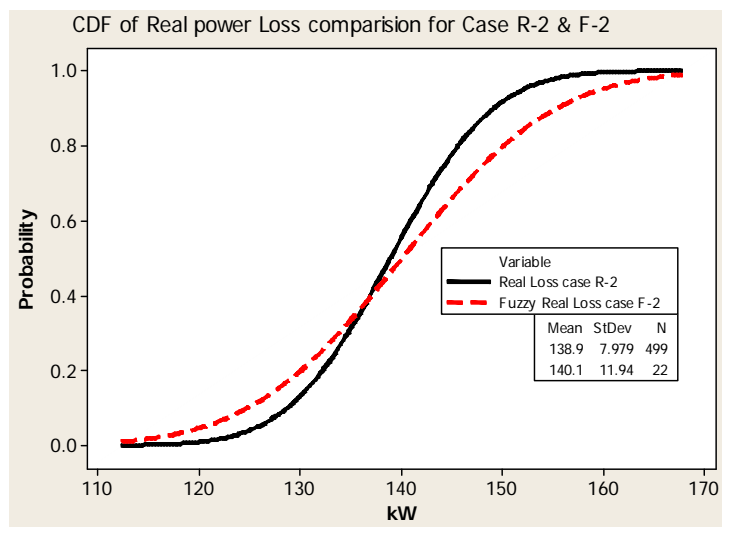

Figure 16. CDF of real power loss comparison for case R-2 \& F-2.
The reason of different variance can be explained with the fact that MCS considers random run for all connected load inputs, where as FAC technique takes two extreme values for all connected load values and envelops results for all extreme scenario together.

Simulation result for three cases shows that results obtained for single or limited input variables (i.e. case-3) are similar, however if an system has more uncertain input variables, MCS technique provides better results.

\section{Conclusions}

Fuzzy logic and probability are different ways of expressing uncertainty. While both fuzzy logic and probability theory can be used to represent subjective belief, fuzzy set theory uses the concept of fuzzy set membership (i.e., how much a variable is in a set), and probability theory uses the concept of subjective probability (i.e., how probable do I think that a variable is in a set). While this distinction is mostly philosophical, the fuzzy-logic-derived possibility measure is inherently different from the probability measure; hence they are not directly equivalent. Probability, which ranges from 0.0 to 1.0 , is used to gauge the likelihood that some particular, well-defined state is or will be the case, under conditions of ignorance or chance. Fuzzy set membership function number, which also ranges from 0.0 to 1.0 , indicates the degree to which an individual case or circumstance belongs to a fuzzy set. Here, values between 0.0 and 1.0 are a result of the inherently imprecise nature of the definition of the fuzzy set, not ignorance or chance.

For the MCS approach large numbers of model runs are required (500 model run considered for this simulation), however FAC approach needed only 20 model runs. This is a drawback for MCS method due to its time consuming characteristic. However, when simulation results are analyzed, result was found comparable, when uncertainty is on one input parameter. The variance increases, with increasing numbers of uncertain input variables. This is due to the fact that MCS considers random run for all connected load inputs, where as FAC technique takes two extreme values for connected load values.

It can be concluded that for more than one uncertain input variable, simulation result indicates that MCS method provides better output results compared to FAC, however takes more time due to number of runs. FAC provides an alternate method to MCS when addressing single or limited input variables and is fast.

\section{REFERENCES}

[1] D. M. Falcao, "Load Estimation in Radial Distribution Systems Using Neural Networks and Fuzzy Set Techniques,” Power Engineering Society Summer Meeting, Vancouver, 15-19 July 2001, pp. 1002-1006. 


\section{doi:10.1109/PESS.2001.970195}

[2] C.-W. Liu, C.-S. Chang and M.-C. Su, "Neuro-Fuzzy Networks for Voltage Security Monitoring Based on Syncronized Phasor Measurements," IEEE Transactions on Power Systems, Vol. 13, No. 2, 1998, pp. 326-332. doi:10.1109/59.667346

[3] I. J. Ramirez-Rosad and J. A. Dominguez-Navarro, "Possibilistic Model Based on Fuzzy Sets for the Multiobjective Optimal Planning of Electric Power Distribution Networks," IEEE Transactions on Power Systems, Vol. 19, No. 4, 2004, pp. 1801-1810. doi:10.1109/TPWRS.2004.835678

[4] V. Kumar and M. Schuhmacher, "Fuzzy Uncertainty Analysis in System Modeling," European Symposium on Computer Aided Chemical Engineering, Vol. 20, 2005, pp. 391396. doi:10.1016/S1570-7946(05)80187-7
[5] A. J. Abebe, V. Guinot and D. P. Solomatine, "Fuzzy Alpha-Cut vs. Monte Carlo Techniques in Assessing Uncertainity in Model Parameters," Proceedings of the 4th International Conference on Hydro Informatics, Iowa City, 23-27 July 2000, pp. 1-8.

[6] M. S. Thomas, R. Ranjan and R. Raina, "Fuzzy Modeled Load Flow Solution for Unbalanced Radialpower Distribution System," Proceedings of the IASTED International Conference, Crete, 22-24 June 2011, pp. 153-159. doi:0.2316/P.2011.714-003

[7] D. Thukram, H. M. W. Banda and J. Jerome, “A Robust Three Phase Power Flow Algorithm for Radial Distribution Systems," Journal of Electrical Power Systems Research, Vol. 50, No. 3, 1999, pp. 227-236. doi:10.1016/S0378-7796(98)00150-3 